\title{
Comparison of two structure-exploiting optimization algorithms for integral quadratic constraints
}

\author{
Ragnar Wallin, Anders Hansson and Lieven Vandenberghe \\ Division of Automatic Control \\ Department of Electrical Engineering \\ Linköpings universitet, SE-581 83 Linköping, Sweden \\ WWW: http://www. control.isy.liu.se \\ E-mail: youremail@isy.liu.se
}

March 7, 2003

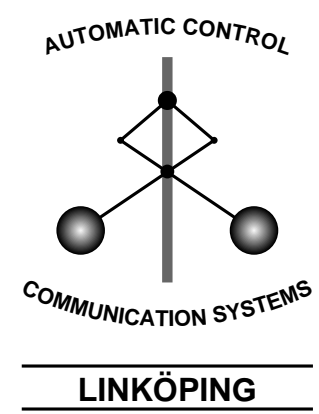

Report no.: LiTH-ISY-R-2502

Submitted to IFAC symposium on robust control design, Milan, Italy, 2003

Technical reports from the Control \& Communication group in Linköping are available at http://www.control.isy.liu.se/publications. 



\title{
Comparison of two structure-exploiting optimization algorithms for integral quadratic constraints
}

\author{
Ragnar Wallin and Anders Hansson \\ Department of Electrical Engineering \\ Division of Automatic Control \\ Linköping University \\ SE-581 83 Linköping, Sweden \\ ragnarw@isy.liu.se, hansson@isy.liu.se \\ Lieven Vandenberghe \\ Electrical Engineering Department \\ University of California, Los Angeles \\ Los Angeles, CA 90095-1594, USA \\ vandenbe@ee.ucla.edu
}

March 7, 2003

\begin{abstract}
As the semidefinite programs that result from integral quadratic constraints are usually large it is important to implement efficient algorithms. The interior-point algorithms in this paper are primal-dual potential reduction methods and handle multiple constraints. Two approaches are made. For the first approach the computational cost is dominated by a least-squares problem that has to be solved in each iteration. The leastsquares problem is solved using an iterative method, namely the conjugate gradient method. The computational effort for the second approach is dominated by forming a linear system of equations. This system of equations is used to compute the search direction in each iteration. If the number of variables are reduced by solving a smaller subproblem the resulting system has a very nice structure and can be solved efficiently. The first approach is more efficient for larger problems but is not as numerically stable.
\end{abstract}

\section{Introduction}

A unified approach to robustness analysis in control using Integral Quadratic Constraints (IQCs) was presented in (Megretski and Rantzer, 1997). Applying the Kalman-Yakubovich-Popov lemma , e. g. (Rantzer, 1996), it can be shown how IQCs can be formulated as Linear Matrix Inequalities (LMIs), e. g. (Boyd et $a l ., 1994 a)$. The corresponding optimization problem is a Semidefinite Program (SDP). 
Efficient interior-point programs for general SDPs have been presented in (Nesterov and Nemirovskii, 1994). These algorithms are still not efficient enough for the large SDPs that result from IQCs. It is, however, possible to increase the efficiency by utilizing the special problem structure inherited from the IQCs.

Previous work on implementing efficient solvers for LMIs originating from IQCs are based on cutting plane algorithms, (Kao et al., 2000) and outer approximation methods, (Parrilo, 1999). Recently, two interior path-following algorithms with frequency dependent barrier functions were presented in (Kao, 2002). Initial results, where the problem structure is exploited, have been presented in (Hansson and Vandenberghe, 2000a), (Hansson and Vandenberghe, 2001), (Wallin et al., 2001) and (Vandenberghe, 2001). In this paper we will expand on the results presented in (Wallin et al., 2001).

\section{Primal and dual problems}

The solution to an IQC problem can be obtained by solving an SDP with the following structure:

$$
\begin{array}{ll}
\operatorname{minimize} & c^{T} x \\
\text { s. t. } & X_{i}=\bar{A}_{i} P_{i} \bar{B}_{i}+\bar{B}_{i}^{T} P_{i} \bar{A}_{i}^{T}+M_{i}(x) \\
& X=\oplus_{i=1}^{N} X_{i} \geq 0 \\
& i=1,2, \ldots, N
\end{array}
$$

where $\bar{A}_{i}^{T}=\left[\begin{array}{ll}I & 0\end{array}\right], \bar{B}_{i}=\left[A_{i} B_{i}\right]$ and $A_{i} \in \mathbf{R}^{n_{i} \times n_{i}}, B_{i} \in \mathbf{R}^{n_{i} \times m_{i}}$. The matrix $M_{i}(x)=M_{i 0}+\sum_{j=1}^{K} x_{j} M_{i j}$ is symmetric. The constraint (1) may be rewritten using linear operators as

$$
X_{i}=M_{0}+\mathcal{F}_{i}\left(P_{i}\right)+\mathcal{G}_{i}(x)
$$

The most efficient algorithms for solving SDPs are primal-dual algorithms. Thus, we will formulate the dual of the SDP (1).

$$
\begin{array}{ll}
\operatorname{maximize} & -\sum_{i=1}^{N}\left\langle Z_{i}, M_{i 0}\right\rangle \\
\text { s. t. } & \bar{B}_{i} Z_{i} \bar{A}_{i}+\bar{A}_{i}^{T} Z_{i} \bar{B}_{i}^{T}=0 \\
& \sum_{i=1}^{N}\left\langle M_{i j}, Z_{i}\right\rangle=c_{j} \\
& Z=\oplus_{i=1}^{N} Z_{i} \geq 0 \\
& i=1,2, \ldots, N ; \quad j=1,2, \ldots, K
\end{array}
$$

The inner product $\langle\cdot, \cdot\rangle$ is the trace of the product of the matrices. The constraints (2) and (3) can be rewritten using adjoint linear operators

$$
\mathcal{F}_{i}^{*}\left(Z_{i}\right)=0 ; \quad \sum_{i=1}^{N} \mathcal{G}_{i}^{*}\left(Z_{i}\right)=c .
$$




\section{Primal-dual potential reduction}

Potential reduction methods start at a strictly feasible point and reduce the potential function

$$
\Phi(X, Z)=\nu \sqrt{n}\langle X, Z\rangle+\log \operatorname{det} X^{-1}+\log \operatorname{det} Z^{-1}+n \log \langle X, Z\rangle-n \log n
$$

by at least a fixed amount in each iteration. The potential function combines the duality gap, $\langle X, Z\rangle$, with the deviation from the central path. The optimal value of the duality gap is known to be zero. The duality gap is an upper bound on the difference between the value of the primal objective and the optimal value of the primal problem. The constant $\nu \geq 1$ sets the relative weight of how important it is to reduce the duality gap and how important it is that the iterate is close to the central path. For more details on how to choose this parameter see e. g. (Vandenberghe and Boyd, 1996). The parameter $n$ is the dimension of $X$. The iterates remain strictly feasible and converge to an optimum in polynomial time. The method works as follows

given strictly feasible $X$ and $Z$.

repeat

1. Find directions $\delta X$ and $\delta Z$. We will use the Nesterov-Todd direction.

2. Find $p, q \in \mathbf{R}$ that minimize $\Phi(X+\delta X, Z+\delta Z)$ doing a plane search.

3. Update $X=X+p \delta X$ and $Z=Z+q \delta Z$.

until $\langle X, Z\rangle \leq \epsilon$.

The primal-dual potential reduction method used is due to Nesterov and Todd, e. g. (Todd et al., 1998), and it is the same method that is used in SP (Vandenberghe and Boyd, 1998).

\subsection{The Nesterov-Todd search direction}

The Nesterov-Todd direction makes the primal direction primal-dual symmetric. Due to the structure of the problem we can work on one constraint at a time. The following steps is a way to compute the Nesterov-Todd direction (Frenk et al., 1999):

- Compute a Cholesky factorization:

$$
X_{i}=R_{i}^{T} R_{i}
$$

The cost for this is $O\left(n_{i}^{3}\right)$.

- Compute an eigenvalue decomposition:

$$
R_{i} Z_{i} R_{i}^{T} U_{i}=U_{i} \Lambda_{i}
$$

The cost for this is $O\left(n_{i}^{3}\right)$ 
- Compute the matrices:

$$
\begin{aligned}
W_{i} & =R_{i}^{T} U_{i} \Lambda_{i}^{-\frac{1}{4}} \\
S_{i} & =W_{i} W_{i}^{T} \\
D_{i} & =\frac{n+\nu \sqrt{n}}{\langle X, Z\rangle} X_{i}-Z_{i}^{-1}=W_{i}\left(\rho \Lambda_{i}^{\frac{1}{2}}-\Lambda_{i}^{-\frac{1}{2}}\right) W_{i}^{T} .
\end{aligned}
$$

The cost for this is $O\left(n_{i}^{3}\right)$

- Solve the linear system of equations:

$$
\begin{aligned}
& -S_{i} \delta Z_{i} S_{i}+\underbrace{\mathcal{F}_{i}\left(\delta P_{i}\right)+\mathcal{G}_{i}(\delta x)}_{\delta X_{i}}=-D_{i} \\
& \mathcal{F}_{i}^{*}\left(\delta Z_{i}\right)=0 \\
& \sum_{i=1}^{N} \mathcal{G}_{i}^{*}\left(\delta Z_{i}\right)=0 .
\end{aligned}
$$

In this part of the algorithm there is much to be gained from an efficient implementation.

- Compute $\delta X_{i}$ as

$$
\delta X_{i}=S_{i} \delta Z_{i} S_{i}-D_{i}
$$

\section{Approach 1: Conjugate Gradient based method}

To reformulate (4), (5) and (6) as a least-squares problem we first define

$$
\begin{aligned}
\delta Z & =\oplus_{i=1}^{N} \delta Z_{i} \\
\delta P & =\oplus_{i=1}^{N} \delta P_{i} \\
W & =\oplus_{i=1}^{N} W_{i} \\
D & =\oplus_{i=1}^{N} D_{i} \\
\mathcal{F}(\delta P) & =\oplus_{i=1}^{N} \mathcal{F}_{i}\left(\delta P_{i}\right) \\
\mathcal{G}(\delta x) & =\oplus_{i=1}^{N} \mathcal{G}_{i}(\delta x) \\
\mathcal{A}(\delta P, \delta x) & =\mathcal{F}(\delta P)+\mathcal{G}(\delta x)
\end{aligned}
$$

If (4) is premultiplied by $W_{i}^{-1}$ and postmultiplied by $W_{i}^{-T}$ and the above definitions are used we can reformulate the problem as

$$
\begin{aligned}
& \underbrace{W^{T} \delta Z W}_{\delta \tilde{Z}}-\underbrace{W^{-1} \mathcal{A}(\delta P, \delta x) W^{-T}}_{\tilde{\mathcal{A}}(\delta P, \delta x)}=W^{-1} D W^{-T} \\
& \underbrace{\mathcal{A}^{*}\left(W^{-T} \delta \tilde{Z} W^{-1}\right)}_{\tilde{\mathcal{A}}^{*}(\delta \tilde{Z})}=0 .
\end{aligned}
$$

This is equivalent to the least-squares problem

$$
\min _{\delta P, \delta x}\left\|\tilde{\mathcal{A}}(\delta P, \delta x)+W^{-1} D W^{-T}\right\|
$$


and

$$
\delta \tilde{Z}=\tilde{\mathcal{A}}(\delta P, \delta x)+W^{-1} D W^{-T}
$$

The normal equations are solved using the conjugate gradient method with a block-Jacobi preconditioner. Utilizing the structure of the problem is in this case the same as chosing an appropriate preconditioner. To make the algorithm efficient it is essential to terminate prior to convergence. Notice that it is sufficient to have $\delta X, \delta Z$ to be a descent direction for the potential function (Vandenberghe and Boyd, 1995), (Boyd et al., 1994b). The computational complexity is experimentally found to be $O\left(\max \left[n_{i}\right]^{3}\right)$. For more details see (Hansson and Vandenberghe, 2000b).

\section{Approach 2: Reducing the number of vari- ables}

In this approach, first introduced in (Vandenberghe, 2001), we try to reduce the number of variables. This is done by calculating basis matrices for $\delta Z_{i}$ that fulfill equation (5) of the Nesterov-Todd search direction. Having these basis matrices it is also possible to eliminate $\delta P_{i}$ from (4). This allows us to solve a much smaller system of equations when we are to calculate the search direction. If we partition $\delta Z_{i}$ as

$$
\delta Z_{i}=\left[\begin{array}{ll}
\delta Z_{i(11)} & \delta Z_{i(12)} \\
\delta Z_{i(12)}^{T} & \delta Z_{i(22)}^{T}
\end{array}\right]
$$

then $\mathcal{F}_{i}^{*}\left(\delta Z_{i}\right)$ can explicitly be written

$$
\mathcal{F}_{i}^{*}\left(\delta Z_{i}\right)=A_{i} \delta Z_{i(11)}+\delta Z_{i(11)} A_{i}^{T}+B_{i} \delta Z_{i(12)}^{T}+\delta Z_{i(12)} B_{i}^{T} .
$$

A basis for $\delta Z_{i}$ that satisfies $\mathcal{F}_{i}^{*}\left(\delta Z_{i}\right)=0$ is

$$
F_{i k}=\left\{\begin{array}{ll}
{\left[\begin{array}{cc}
E_{i k(11)} & E_{i k(12)} \\
E_{i k(12)}^{T} & 0
\end{array}\right]} & k=1,2, \ldots, m_{i} n_{i} \\
{\left[\begin{array}{cc}
0 & 0 \\
0 & E_{i k(22)}
\end{array}\right]} & k=m_{i} n_{i}+1, \ldots, k_{i_{\max }}
\end{array} .\right.
$$

where $k_{i_{\max }}=m_{i} n_{i}+\frac{m_{i}\left(m_{I}+1\right)}{2}, E_{i k(12)}$ is the standard basis for unstructured $n_{i} \times m_{i}$ matrices and where $E_{i k(22)}$ is the standard basis for symmetric $m_{i} \times m_{i}$ matrices. The matrix $E_{i k(11)}$ is related to $E_{k(12)}$ through (5), that is

$$
A_{i} E_{i k(11)}+E_{i k(11)} A_{i}^{T}+B_{i} E_{i k(12)}^{T}+E_{i k(12)} B_{i}^{T}=0 .
$$

We can express $\delta Z_{i}$ as

$$
\delta Z=\sum_{k=1}^{k_{i_{\max }}} \delta z_{i k} F_{i k} .
$$


The cost for finding the basis is $O\left(\max \left[n_{i}\right]^{4}\right)$. In case the $A_{i}$ s are diagonal or can be made diagonal the cost is, however, only $O\left(\max \left[n_{i}\right]^{3}\right)$. Observe that the basis matrices only have to be computed once and that this can be done in advance. Every basis matrix satisfies (5) and thus

$$
\left\langle F_{i k}, \mathcal{F}_{i}\left(\delta P_{i}\right)\right\rangle=\langle\underbrace{\mathcal{F}_{i}^{*}\left(F_{i k}\right)}_{=0}, \delta P_{i}\rangle=0, \quad \forall \delta P_{i} .
$$

This fact can be used to eliminate $\delta P_{i}$ from (4). If we take the inner product between $F_{i j}$ and (4) we get

$$
\begin{aligned}
& -\left\langle F_{i j}, S_{i} \delta Z_{i} S_{i}\right\rangle+\underbrace{\left\langle F_{i j}, \mathcal{F}_{i}\left(\delta P_{i}\right)\right\rangle}_{=0}+\left\langle F_{i j}, \mathcal{G}_{i}(\delta x)\right\rangle \\
& =-\sum_{k=1}^{m_{i} n_{i}+\frac{m_{i}\left(m_{i}+1\right)}{2}} \delta z_{i k}\left\langle F_{i j}, S_{i} F_{i k} S_{i}\right\rangle+\sum_{k=1}^{K} \delta x_{k}\left\langle F_{i j}, M_{i k}\right\rangle=-\left\langle F_{i j}, D_{i}\right\rangle
\end{aligned}
$$

This allows us to rewrite (4) and (6) on the form

$$
\left[\begin{array}{ccccc}
-H_{1} & 0 & \ldots & 0 & G_{1} \\
0 & -H_{2} & \cdots & 0 & G_{2} \\
\vdots & \vdots & \ddots & \vdots & \vdots \\
0 & 0 & \cdots & -H_{N} & G_{N} \\
G_{1}^{T} & G_{2}^{T} & \cdots & G_{N}^{T} & 0
\end{array}\right]\left[\begin{array}{c}
\delta z_{1} \\
\delta z_{2} \\
\vdots \\
\delta z_{N} \\
\delta x
\end{array}\right]=\left[\begin{array}{c}
g_{1} \\
g_{2} \\
\vdots \\
g_{N} \\
0
\end{array}\right]
$$

where $\delta z_{i}$ is a vector with all the unknown $\delta z_{i k}$ stacked on top of each other and $\delta x$ is a vector with all the unknown $\delta x_{j}$. The first block rows correspond to (4), the last block row corresponds to (6). Each element of a block $H_{i}$ is

$$
H_{i(j k)}=\left\langle F_{i j}, S_{i} F_{i k} S_{i}\right\rangle
$$

and the elements of the blocks $G_{i}$ and $g_{i}$ are

$$
\begin{aligned}
& G_{i(j k)}=\left\langle F_{i j}, M_{i k}\right\rangle \\
& g_{i j}=-\left\langle F_{i j}, D_{i}\right\rangle .
\end{aligned}
$$

Note, as $G_{i}$ does not depend on the current iterate it can be computed beforehand. The $H_{i}$ s are positive definite as

$$
\begin{aligned}
x^{T} H_{i} x & =\left\langle\sum_{j} \sum_{k} x_{j} x_{k} F_{i j} S_{i} F_{i k} S_{i}, I\right\rangle \\
& =\left\langle\sum_{j} x_{j} S_{i}^{\frac{1}{2}} F_{i j} S_{i}^{\frac{1}{2}}, \sum_{k} x_{k} S_{i}^{\frac{1}{2}} F_{i k} S_{i}^{\frac{1}{2}}\right\rangle \\
& =\left\|\sum_{k} x_{k} S_{i}^{\frac{1}{2}} F_{i k} S_{i}^{\frac{1}{2}}\right\|_{F}^{2} .
\end{aligned}
$$

The block arrow system (8) with positive definite $H_{i}$ s can be solved efficiently. The dominating cost for finding the search direction is the computation of the $H_{i}$ s. This cost is $O\left(\max \left[n_{i}\right]^{4}\right)$ and is actually larger than the cost for solving 
the system (8). The search direction $\delta X_{i}$ is evaluated as in (7). Once the algorithm has converged the $P_{i}$ s can be computed by solving the Lyapunov equation corresponding to the $(1,1)$-block of $(1)$.

It is tempting to further reduce the number of variables by finding a basis that fulfills both (5) and (6). This can be done for example by computing a QR-factorization of $G$ (Nocedal and Wright, 1999) and would result in a system

$$
-\tilde{H} \delta \tilde{z}=\tilde{g}
$$

where $\tilde{H}$ is slightly smaller in size than the blockdiagonal $H$ of (8) but has no structure at all. Hence, we cannot solve this system of equations as efficiently as $(8)$.

\section{Practical aspects}

As mentioned before the algorithms in this paper are feasible algorithms, i. e. every iterate is strictly feasible. It is usually not trivial to find a strictly feasible initial point. We have chosen to use a two phased method to get around this difficulty. In the first phase we have the primal problem

$$
\begin{aligned}
& \operatorname{minimize} \bar{M} t \\
& \text { s. t. } X_{i}=\bar{A}_{i} P_{i} \bar{B}_{i}+\bar{B}_{i}^{T} P_{i} \bar{A}_{i}^{T}+M_{i}(x)+t I \\
& X=\oplus_{i=1}^{N} X_{i} \geq 0 \\
& x_{j}+\overline{\bar{M}} \geq 0 \\
& -x_{j}+\overline{\bar{M}} \geq 0 \\
& t+\alpha \geq 0 \\
& i=1,2, \ldots, N
\end{aligned}
$$

The artificial constraints on the $x_{j}$ s makes it trivial to find a feasible point for the dual problem. The restrictions on the $x_{j}$ s means that we are only looking in a hypercube. If we can find a feasible solution to this problem with a negative primal objective function we have found a feasible primal point to the original primal problem (1) and can proceed to the second phase. On the other hand, if we find a feasible solution with a positive dual objective function there is no strictly feasible solution inside the hypercube. Of course, we may always try a larger value of $\bar{M}$. In the second phase we use the $P_{i}$ s and the $x_{j}$ s that we got from the first phase as initial points and have the primal problem

$$
\begin{array}{ll}
\operatorname{minimize} & c^{T} x \\
\text { s. t. } & X_{i}=\bar{A}_{i} P_{i} \bar{B}_{i}+\bar{B}_{i}^{T} P_{i} \bar{A}_{i}^{T}+M_{i}(x) \\
& X=\oplus_{i=1}^{N} X_{i} \geq 0 \\
& x_{j}+\overline{\bar{M}} \geq 0 \\
& -x_{j}+\overline{\bar{M}} \geq 0 \\
& i=1,2, \ldots, N
\end{array}
$$

Again, the artificial bounds on the $x_{j}$ s makes it trivial to find a feasible point to the dual problem. The optimal solution to this SDP is also an optimal solution to (1), provided the constraints involving $\bar{M}$ are not active. In case the constraints are active we may always try a larger value of $\overline{\bar{M}}$. 


\section{Numerical examples}

\section{Example 1}

This is the same example as Example 1 in (Parrilo, 1999). Consider the block diagram in Figure 1. We want to compute the worst case $\mathcal{L}_{2}$ induced norm between $u$ and $y$.

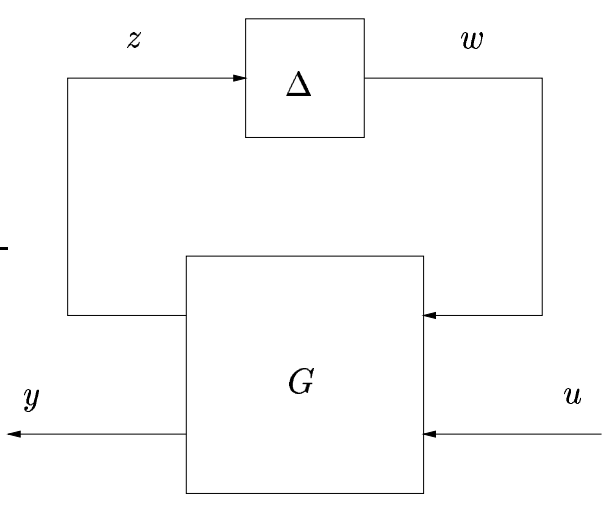

Figure 1: Block diagram for Example 1.

The plant $G$ is given by

$$
G(s)=\left[\begin{array}{cc}
\frac{s+1}{s^{2}+2 s+2} & 1 \\
1 & 0
\end{array}\right]
$$

The $\Delta$-block is an uncertain contractive linear time-varying operator. It can be shown that finding the the worst case $\mathcal{L}_{2}$ induced norm is equivalent to solving the SDP

$$
\begin{array}{ll}
\operatorname{minimize} & x_{1} \\
\text { s. t. } & X_{1}=\bar{A}_{1} P_{1} \bar{B}_{1}+\bar{B}_{1}^{T} P_{1} \bar{A}_{1}^{T}+M_{1}(x) \\
& X_{2}=M_{2}(x) \\
& X=X_{1} \oplus X_{2} \geq 0 \\
& P_{1} \in \mathbf{R}^{2 \times 2} \quad \text { and } \quad x \in \mathbf{R}^{2}
\end{array}
$$

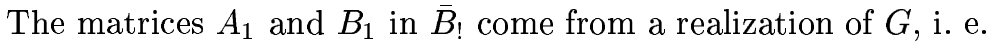

$$
\left[\begin{array}{l}
\dot{\xi}(t) \\
z(t) \\
y(t)
\end{array}\right]=\left[\begin{array}{ll}
A_{1} & B_{1} \\
C_{1} & D_{1}
\end{array}\right]\left[\begin{array}{c}
\xi(t) \\
w(t) \\
u(t)
\end{array}\right] .
$$


The matrices $M_{i j}$ are defined such that

$$
\begin{aligned}
& {\left[\begin{array}{l}
\xi(t) \\
w(t) \\
u(t)
\end{array}\right]^{T} M_{10}\left[\begin{array}{l}
\xi(t) \\
w(t) \\
u(t)
\end{array}\right]=-y^{2}(t)} \\
& {\left[\begin{array}{l}
\xi(t) \\
w(t) \\
u(t)
\end{array}\right]^{T} M_{11}\left[\begin{array}{l}
\xi(t) \\
w(t) \\
u(t)
\end{array}\right]=u^{2}(t)} \\
& {\left[\begin{array}{l}
\xi(t) \\
w(t) \\
u(t)
\end{array}\right]^{T} M_{12}\left[\begin{array}{l}
\xi(t) \\
w(t) \\
u(t)
\end{array}\right]=w^{2}(t)-z^{2}(t)} \\
& M_{20}=0 \\
& M_{21}=0 \\
& M_{22}=1
\end{aligned}
$$

The solution is given by

$$
P=-\left[\begin{array}{ll}
3.495 & 0.667 \\
0.667 & 0.667
\end{array}\right] \quad x=\left[\begin{array}{l}
7.548 \\
2.747
\end{array}\right]
$$

We ran the example for different choices of $\nu$ and $\overline{\bar{M}}$. The results for the second phase are given in Table 1 . The conjugate gradient approach is very sensitive to the choice of $\nu$ and $\overline{\bar{M}}$ in the sense that the number of iterations for the conjugate gradient iterates and the number of outer iterates vary very much depending on those parameters. The elimination of variables approach, on the other hand, is rather robust to the choice of $\nu$ and $\bar{M}$. It is very unfortunate to have a method that is sensitive to $\bar{M}$ as the numeric value of this parameter is dependent on the problem. $\overline{\bar{M}}$ has to be greater than the maximum absolute value of all $x_{j}$. Otherwise the optimal $x$ is not in the hypercube.

\begin{tabular}{|r|r|r|r|r|r|}
\hline$\nu$ & $\bar{M}$ & \# iter CG & CPU time CG [s] & \# iter ELIM & CPU time ELIM [s] \\
\hline 3 & 10 & 16 & 11.68 & 8 & 0.45 \\
\hline 3 & 100 & 32 & 41.56 & 11 & 0.53 \\
\hline 3 & 1000 & 29 & 41.23 & 13 & 0.76 \\
\hline 10 & 10 & 10 & 2.42 & 5 & 0.29 \\
\hline 10 & 100 & 26 & 17.16 & 8 & 0.49 \\
\hline 10 & 1000 & 35 & 46.47 & 9 & 0.51 \\
\hline 30 & 10 & 15 & 4.00 & 6 & 0.33 \\
\hline 30 & 100 & 16 & 20.89 & 7 & 0.42 \\
\hline 30 & 1000 & 30 & 20.69 & 8 & 0.46 \\
\hline
\end{tabular}

Table 1: Computational results for Example 1. The columns labeled CG are for the conjugate gradient based approach and the columns labeled ELIM are for the elimination of variables approach.

\section{Example 2}

The setup in this example is the same as in Example 1 above except that $u, w$, $y$, and $z$ are vector-valued, and that the $\Delta$-block is a diagonal contractive linear 
time-varying operator. The derivation is similar, but there are now instead as many IQCs associated with the $\Delta$-block as the dimension $\bar{p}$ of $w$ or equivalently $z$. This is the same example as Example 2 in (Parrilo, 1999) except for the numerical values.

For simplicity we will assume that $u$ and $y$ have the same dimensions as $w$ and $z$, i.e. $\bar{p}$. We construct random systems in the following way. Let $\lambda_{i j}, j=1,2, \ldots, 4, i=1,2, \ldots, a$, be drawn from a uniform distribution on $[-2,-1]$, and define

$$
\tilde{A}_{i}=\operatorname{diag}_{j}\left(\lambda_{i j}\right)+\tilde{A}_{0} \in \mathbf{R}^{4 \times 4}
$$

where $\tilde{A}_{0}$ is a matrix of zeros except for the first super-diagonal which have all elements equal to one. Then let $\tilde{A}=\operatorname{blockdiag}_{i}\left(\tilde{A}_{i}\right)$. Similarly define

$$
\tilde{B}_{i}=\left[\begin{array}{c}
0 \\
\vdots \\
0 \\
1
\end{array}\right] \in \mathbf{R}^{4 \times 1}
$$

and let $B_{w}=B_{u}=\operatorname{blockdiag}_{i}\left(\tilde{B}_{i}\right)$, and $\tilde{B}=\left[\begin{array}{ll}B_{w} & B_{u}\end{array}\right]$. For $\tilde{C}$ introduce

$$
\tilde{C}_{i}=\left[\begin{array}{llll}
1 & 0 & \cdots & 0
\end{array}\right] \in \mathbf{R}^{1 \times 4}
$$

and let $C_{y}=C_{z}=\operatorname{blockdiag}_{i}\left(\tilde{C}_{i}\right)$, and $\tilde{C}^{T}=\left[\begin{array}{ll}C_{z}^{T} & C_{y}^{T}\end{array}\right]$. The dimensions of $(\tilde{A}, \tilde{B}, \tilde{C})$ are $n_{1} \times n_{1}, n_{1} \times m_{1}$ and $m_{1} \times n_{1}$, respectively, where $m_{1}=2 \bar{p}$ and $n_{1}=\sum_{i=1}^{\bar{p}} 4$. In order to couple all inputs with all outputs we will introduce random unitary state, input, and output transformations. To this end let $\bar{S}_{n_{1}} \in$ $\mathbf{R}^{n_{1} \times n_{1}}$ and $\bar{S}_{m_{1}} \in \mathbf{R}^{m_{1} \times m_{1}}$ be matrices with entries drawn from a uniform distribution on $[0,1]$. Let the singular value decompositions of these matrices be given by

$$
\bar{S}_{n_{1}}=U_{n_{1}} \Sigma_{n_{1}} V_{n_{1}}^{T} ; \quad \bar{S}_{m_{1}}=U_{m_{1}} \Sigma_{m_{1}} V_{m_{1}}^{T}
$$

and define

$$
A_{1}=U_{n_{1}}^{T} \tilde{A} U_{n_{1}} ; B_{1}=U_{n_{1}}^{T} \tilde{B} V_{m_{1}} ; C_{1}=U_{m_{1}} \tilde{C} U_{n_{1}}
$$

Now we let $G$ be such that it has $\left(A_{1}, B_{1}, C_{1}\right)$ as a realization. The matrices $M_{i j}, i=1,2, \ldots, p$, where $p=1+\bar{p}$, are defined similarly as for Example 1. The computational results for the second phase are shown in Table 2. Here, $\nu=3$ and the $\bar{M}=10$. This choice made the conjugate gradient approach perform well. It is obvious that the conjugate gradient approach is more efficient than the elimination of variables approach for large problems. However, the parameter $\bar{M}$ and the parameter $\nu$ have to be well chosen if the conjugate gradient based approach is going to perform well.

\section{Conclusions and future work}

In this paper was presented two approaches to efficiently solve SDPs originating from IQCs. In the conjugate gradient based method the number of iterations is highly dependent on the parameter $\nu$, which weights how important it is to 


\begin{tabular}{|l|r|r|r|r|r|r|}
\hline$a$ & 1 & 2 & 3 & 4 & 5 & 6 \\
\hline$n$ & 29 & 33 & 37 & 41 & 45 & 49 \\
\hline \# variables & 105 & 189 & 305 & 453 & 633 & 845 \\
\hline CPU time CG [s] & 11.24 & 11.39 & 12.10 & 16.35 & 29.99 & 39.85 \\
\hline CPU time ELIM [s] & 12.09 & 25.48 & 50.53 & 97.27 & 173.10 & 293.07 \\
\hline
\end{tabular}

Table 2: Computational results for Example 2. The columns labeled CG are for the conjugate gradient based approach and the columns labeled ELIM are for the elimination of variables approach.

decrease the duality gap to how important it is to stay close to the central path, and the choice of $\bar{M}$. For large problems this approach is more efficient.

The number of iterations to decrease the duality gap to a certain value is always smaller for the elimination of variables approach. This approach is also more reliable. Furthermore, there is potential to increase the efficiency of this method, using special structure of the $A_{i}$ s and computing the matrix $H$ in (8) using the fast Fourier transform (Vandenberghe, 2001).

\section{Acknowledgement}

We are very grateful to Per Rehnquist, Department of Signals, Sensors and systems, Royal Institute of Technology for his work on the conjugate gradient solver. We acknowledge financial support from the Swedish Research Council under grant 271-2000-770.

\section{References}

Boyd, S., L. El Ghaoui, E. Feron and V. Balakrishnan (1994a). Linear matrix inequalities in system and control theory. SIAM. Philadelphia, USA.

Boyd, S., L. Vandenberghe and M. Grant (1994b). Efficient convex optimization for engineering design. In: Proceedings of the IFAC symposium on robust control design. Rio de Janeiro, Brazil. pp. 14-23.

Frenk, H., K.Roos, Terlaky, T. and Zhang, S., Eds.) (1999). High performance optimization. Chap. Symmetric primal-dual transformations, pp. 61-91. Kluwer Academic Publishers. Dordrecht, The Netherlands.

Hansson, A. and L. Vandenberghe $(2000 a)$. Efficient solution of linear matrix inequalities for integral quadratic constraints. In: Proceedings of the 39th IEEE Conference on Decision and Control. Vol. 5. Sydney, Australia. pp. 5033-5034.

Hansson, A. and L. Vandenberghe (2000b). Efficient solution of linear matrix inequalities for integral quadratic constraints. Internal Report IR-S3-REG0002. Division of Automatic Control. Royal Institute of Technology, Stockholm. 
Hansson, A. and L. Vandenberghe (2001). A primal-dual potential reduction method for integral quadratic constraints. In: Proceedings of the American Control Conference. Arlington, Virginia, USA. pp. 3013-3017.

Kao, C. Y., A. Megretski and U. T. Jönsson (2000). An algorithm for solving optimization problems involving special frequency dependent LMIs. In: Proceedings of the 2000 American Control Conference. Vol. 1. Chicago, Illinois, USA. pp. 307-311.

Kao, C.Y. (2002). Efficient computational methods for robustness analysis. PhD thesis. Massachusetts Institute of Technology.

Megretski, A. and A. Rantzer (1997). System analysis via integral quadratic constraints. IEEE Transactions on Automatic Control AC-42(6), 819-830.

Nesterov, Y. and A. Nemirovskii (1994). Interior point polynomial algorithms in convex programming. SIAM. Philadelphia, USA.

Nocedal, J. and S.J. Wright (1999). Numerical Optimization. Springer Verlag. New York, USA.

Parrilo, P. A. (1999). On the numerical solution of LMIs derived from the KYP lemma. In: Proceedings of the 38th IEEE Conference on Decision and Control. Vol. 3. Phoenix, Arizona, USA. pp. 2334-2338.

Rantzer, A. (1996). On the Kalman-Yakubovich-Popov lemma. Systems $\&$ Control Letters 28(1), 7-10.

Todd, M. J., K. C. Toh and R. H. Tütüncü (1998). On the Nesterov-Todd direction in semidefinite programming. SIAM Journal on Optimization 8(3), 769-796.

Vandenberghe, L. (2001). Interior-point methods for semidefinite programming problems in signal processing and control. In: Fourth SIAM conference on linear algebra in signals, systems and control. Boston, Massachusetts, USA. Abstract only.

Vandenberghe, L. and S. Boyd (1995). A primal-dual potential reduction method for problems involving matrix inequalities. Mathematical Programming 69(1, Ser. B), 205-236.

Vandenberghe, L. and S. Boyd (1996). Semidefinite programming. SIAM Review 38(1), 49-95.

Vandenberghe, L. and S. Boyd (1998). SP-Software for Semidefinite Programming-Users Guide. University of California Los Angeles and Stanford University.

Wallin, R., H. Hansson and L. Vandenberghe (2001). Efficient implementations of interior-point methods for integral quadratic constraints. In: Fourth SIAM conference on linear algebra in signals, systems and control. Boston, Massachusetts, USA. Abstract only. 